\title{
Spatial distance in a technology gap model
}

\author{
by \\ M.C.J. Caniëls (TUE) and B. Verspagen (ECIS and MERIT)
}

May 1999

\begin{abstract}
This paper analyses the effect of locally bounded knowledge spillovers on regional differences in growth. A model will be developed that allows spillovers to take place across regions. Certain conditions determine the amount of spillovers a region receives. By use of simulations (with randomised parameter constellations), it is possible to examine the general behaviour of the model. It is found that certain patterns in the gaps of the knowledge stocks appear repeatedly. The inclusion of geographical distance in a technology gap model leads to the observation that when differences (in parameters such as the learning capability or the exogenous rate of knowledge generation) increase across regions, the disparity in terms of GDP per capita between them decreases. This counterintuitive result is a direct effect from the inclusion of geographical distance in the model.
\end{abstract}




\section{Introduction}

The issue of convergence of GDP per capita is the topic of a large and growing literature in economics (e.g., Barro and Sala-i-Martin, 1995, Fagerberg, 1994). The general conclusion from this literature is that convergence, as opposed to divergence, is a special outcome that may prevail between a set of countries that is relatively homogenous in terms of variables such as knowledge generation (R\&D), infrastructure, educational systems, etc.

This relationship between homogeneity of 'structural' characteristics and convergence arises from at least two different theoretical perspectives. On the one hand, there is the notion of 'conditional convergence' that arises from new growth models in the neoclassical tradition (e.g., Barro and Sala-i-Martin, 1995). Here, the basic idea is that long-run economic growth can be described by a steady state balanced path. In the short run, countries that have not yet reached their steady state show higher (or lower, depending on which side they are approaching the steady state from) growth than countries closer to the steady state. If steady states are similar between countries, convergence is unconditional, but when, as in the new models, steady states differ, convergence becomes conditional upon variables such as R\&D and infrastructure. In the latter case of heterogeneity, observed empirical patterns of growth may show little or no convergence. The more homogenous the group of countries under consideration becomes, however, the more convergence becomes likely.

The other theoretical perspective on convergence emerges from so-called technology gap theory (e.g., Fagerberg, 1994). Here convergence results from the international diffusion of knowledge. Initially backward countries benefit from the inflow of knowledge that was developed in the more advanced countries. However, the extent to which they are able to benefit from this depends on so-called social capability and technological congruence (Abramovitz, 1994). Social capability refers to variables that are largely similar to the variables defining the steady state in neoclassical models, such as education and infrastructure. Technological congruence refers, for example, to the match between the sectoral structure in the backward and advanced countries. When technological congruence is large, and social capabilities do not differ too much between countries, convergence will become more likely.

One aspect that remains relatively obscured in both theoretical perspectives on convergence, is the impact of spatial proximity on the diffusion of technological knowledge. The models in the technology gap tradition (e.g., Fagerberg, 1988, Verspagen, 1991, Amable, 1993) do not take into account spatial proximity as a determinant of knowledge diffusion. Nevertheless, there is a large literature in economic geography that underlines the importance of proximity for knowledge spillovers. The concept of interest in this literature (for an overview see Baptista, 1998) is the existence of agglomeration economies and its effects on growth. Agglomeration economics involve the positive effects on a firm or a region generated by a spatial concentration of economic activity. Agglomeration economies are induced, among others, by a large opportunity for communication of ideas and experience, which is enhanced by spatial proximity. In this paper we focus on knowledge spillovers as the prime form of agglomeration economies. Several studies (e.g., Acs, Audretsch and Feldman, 1992, 
Jaffe, Trajtenberg and Henderson, 1993) have confirmed such a positive relation between geographic proximity and knowledge spillovers.

Theoretical reasons for the localized nature of knowledge spillovers are as follows. Technological knowledge is often informal, tacit and uncodified in its nature (e.g., Pavitt, 1987). This implies that there are differences between knowledge and information, where the former concept is more far-reaching than the latter. Audretsch and Feldman (1996) argue that although the cost of transmitting information may be invariant to distance, presumably the cost of transmitting knowledge rises with distance. Possibilities for learning-by-doing and learning-by-using, important for the transmission of knowledge, to a large extent come from direct contacts with competitors, customers, suppliers and providers of services (Von Hippel, 1988, 1994) and are therefore highly dependent on proximity.

Uncertainty is another characteristic of the innovative process. Interaction between innovators, e.g. in regional networks, helps to reduce this uncertainty. This kind of interaction is highly dependent on geographical proximity. In this respect, Freeman (1991) points out that networks frequently tend to be localised. Another reason why proximity has an effect on the innovative process lies in the fact that innovation relies heavily upon sources of basic scientific knowledge. Jaffe (1989) and Acs, Audretsch and Feldman (1992) have empirically shown that knowledge spillovers from university research to private firms are facilitated by geographic proximity. Furthermore, innovative activity is cumulative, meaning that new innovations build upon scientific knowledge generated by previous innovations. Breschi (1995) and Malerba and Orsenigo (1995) point out that the accumulation of innovative activity in a geographic area facilitates the generation of new innovations in this area.

The aim of this paper is to integrate space into a model of technology gaps, and to investigate whether or not the conclusion of a relationship between homogeneity of countries and convergence remains in tact. In order to do so, we incorporate spatial proximity into a technology gap growth model developed earlier by Verspagen (1991). The resulting model is one in which a multitude of geographic units (which will be called regions) interact with each other in terms of knowledge diffusion. These regions may differ with respect to their R\&D efforts and their social capability to assimilate knowledge from other regions. Ceteris paribus, knowledge from regions close by diffuses more easily than knowledge from regions far away.

The rest of this paper is organised as follows. In Section 2, the part of the model that describes technological spillovers is first presented for the case of two regions. Later on, this is extended to a multi-region model. Section 3 explores the outcomes of our model in terms of patterns of convergence or divergence by means of simulation techniques. Finally, Section 4 summarises the main conclusions from this paper.

\section{Description of the spillover system}

For simplicity, we disregard any sources of output growth other than the growth of technological knowledge. Specifically, it is assumed that output growth is a linear function of the growth of the knowledge stock: 


$$
\frac{\dot{Q}_{i}}{Q_{i}}=\beta \frac{\dot{K}_{i}}{K_{i}},
$$

in which $Q_{i}$ denotes the level of output of region $i$ and $K_{i}$ points to the level of the knowledge stock of region $i$. $\beta$ is a parameter, indicating the proportion of the knowledge stock growth that results in output growth. Dots above variables denote time derivatives.

New knowledge is assumed to stem from three sources: learning-by-doing (modelled as a Verdoorn effect ${ }^{1}$ ), spillovers received from surrounding (not necessarily contingent) regions $\left(S_{i}\right)$, and an exogenous rate of growth $\left(\rho_{i}\right)$, which can be thought of as reflecting the impact of exogenous $R \& D$ activities in the region. This yields the following equation:

$$
\frac{\dot{K}_{i}}{K_{i}}=\alpha\left(\lambda \frac{\dot{Q}_{i}}{Q_{i}}+S_{i}+\rho_{i}\right),
$$

in which $\alpha$ and $\lambda$ are parameters. $\alpha$ points out the extent to which the knowledge stock growth is influenced by the above factors, and $\lambda$ reflects the intensity of the Verdoorn effect.

For the explanation of the spillover term $S$, it is convenient to first consider two regions, later on this framework will be extended, and a multi-region model will be constructed. In the two-region setting, it is assumed that there is one technologically advanced region and one backward region. Spillovers depend on the size of the knowledge gap, as well as three different parameters reflecting distinct effects related to the realisation of potential spillovers. We use the following equation to model spillovers:

$$
\begin{gathered}
S_{i}=\frac{\delta_{i}}{\gamma_{i j}} e^{-\left(\frac{1}{\delta_{i}} G_{i j}-\mu_{i}\right)^{2}}, \\
G_{i j}=\ln \frac{K_{i}}{K_{j}},
\end{gathered}
$$

in which $S_{i}$ denotes the spillovers generated by region $j$ and received by region $i^{2} . G_{i j}$ denotes the technology gap of region $i$ towards region $j$, and is defined as the log of the ratio of the knowledge stocks of two regions. The realisation of the potential spillover level depends on the three parameters $\gamma, \delta$ and $\mu$, which we will now discuss in turn.

$\gamma_{i j}$ is the geographical distance between two regions. If $\gamma_{i j}$ increases, the spillover is reduced. This assumption stems from the geographical literature. As was discussed in the

\footnotetext{
${ }^{1}$ The Verdoorn-Kaldor law states that a positive relation exists between the growth of productivity and the growth of output.

${ }^{2}$ Note that the lower the initial stock of knowledge a region is endowed with, the more spillovers it will receive. This is similar to the concept of $\beta$-convergence (Barro, 1984; Baumol, 1986; De Long, 1988; Barro and Sala-iMartin, 1991, 1992a, 1992b) in which a backward economy (an economy with a low initial level of GDP per capita) will grow faster than a rich economy and therefore catch up.
} 
introduction of this paper, this is based on the assumption that spatial proximity eases spillovers (agglomeration economies), because interaction between the receiver and generator of the spillovers is easier when distance is small. $\mu_{\mathrm{i}}$ and $\delta_{\mathrm{i}}$ are two parameters that are related to the intrinsic learning capability of region $i$. These parameters thus reflect the broad concept of 'social capability' to assimilate spillovers (e.g., Abramovitz, 1994). Regions that have a high social capability to learn (e.g., a highly educated workforce, good infrastructure, an efficient financial system, etc.), can implement the knowledge from other regions more easily. $\mu_{\mathrm{i}}$ and $\delta_{\mathrm{i}}$ reflect different parts of the learning capability that will be explained further by means of graphical analysis.

Figure 1 presents the spillover functions for two regions, assuming all parameters to be equal between the regions. The horizontal axis displays the size of the technology gap. Note that a positive value of $G_{i j}$ by definition implies that region $j$ is the backward region. A first

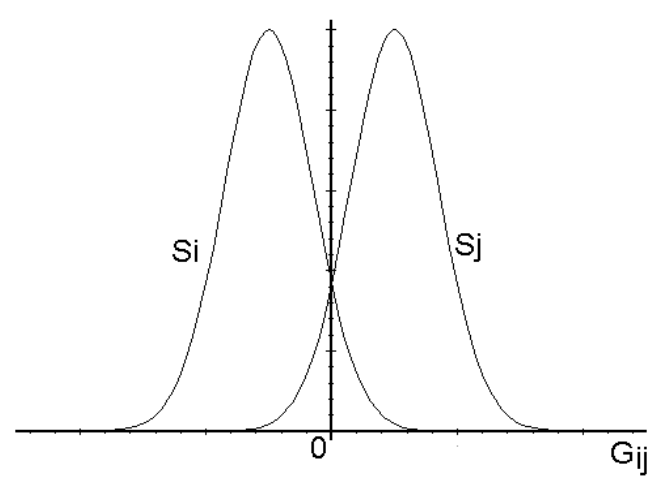

Figure 1

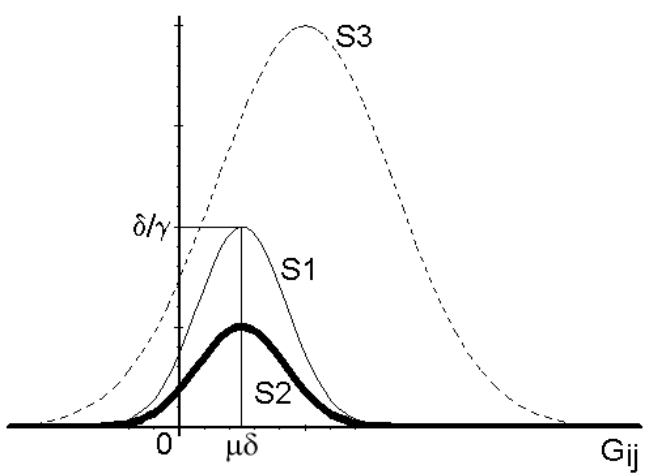

Figure 2

characteristic of our model is that, in contrast with most of the catch-up literature, we allow spillovers to occur in two directions, i.e., from the technological leader to backward region(s), and vice versa. Figure 1 shows that, for equal parameter values between the regions, the spillover stream from the advanced to the backward region will be larger than the reverse stream ( $S_{i}$ is always below $S_{j}$ to the right of the vertical axis). This reflects the notion that the backward region can learn more than the advanced region. However, spillovers from a backward region to the leader region also take place because it could well be possible that the backward region has (developed) complementary knowledge, knowledge that was not yet in the hands of the leader. So there always is a small flow of knowledge from laggard to leader, although this quantity quickly goes to zero for large gaps.

The net spillover will be equal to zero when the gap between the two regions is zero (i.e., they have equal knowledge stocks). In this situation there are still spillovers, but these are of equal size in both directions. This only holds, however, when the parameters $(\rho, \lambda, \mu, \delta)$ are equal between the two regions. In the more general case of unequal parameters between regions, net spillovers may be positive or negative for a gap of value zero.

Figure 2 displays the spillovers received by one region for this two-region model. Note that the top of each spillover curve lies at a technology gap equal to $\mu_{\mathrm{j}} \delta_{\mathrm{j}}$. The maximal spillover corresponding to this is equal to $\delta_{\mathrm{j}} / \gamma_{\mathrm{ij}}$. We take the curve labelled S1 as the starting point, and 
we consider what happens to the spillover function under certain conditions. First, an enlargement of the geographical distance between two regions (higher $\gamma$ ) will lead to lower spillovers received by each region, depicted by the thick line S2. Note that an increase in distance shifts the curve down, but leaves the value of the gap for which spillovers are maximal unchanged.

Second, an increase in the learning capability parameter $\delta$ of the lagging region will cause the spillover function to shift up, and the maximum of the curve to shift to the right (dotted line S3). ${ }^{3}$ Thus, with higher $\delta$, the laggard is able to learn more (magnitude of the spillover function) and more easily, or earlier (at a larger technological distance).

As will be explained below, the value of $G$ at which the spillover curve peaks $(\mu \delta)$ is important for the result in terms of convergence or divergence. We therefore want to allow for the possibility that maximum of the spillover curve shifts left or right, without affecting the value of the maximum itself. Figure 3 shows how the parameter $\mu$ does exactly this.

If $\mu_{\mathrm{j}}$ is increased, all other things being equal, the curve will shift to the right (S5). This has several effects. First, the level of spillovers in the case of equal knowledge stocks across regions $(G=0)$, is smaller. This indicates that for relatively large $\mu$, the model resembles a regular catch-up model, which is characterised by zero spillovers for zero technological distance. Second, because the top of the curve moves to the right, catch-up becomes easier. At a larger technological distance, it is still possible to catch up. How the distinction between catching-up of falling behind works exactly will become clearer after we discuss the net spillover function.

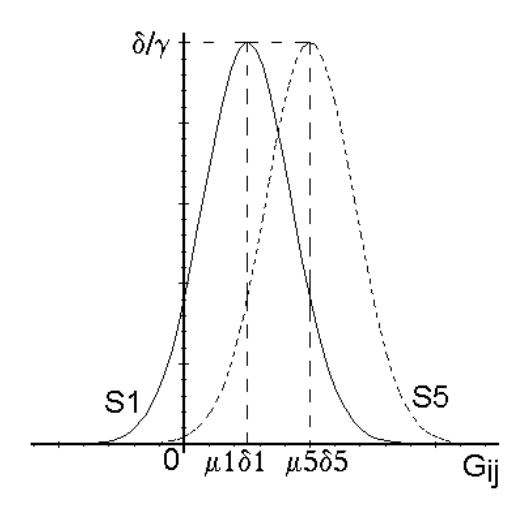

Figure 3

Thus, the difference between the parameters $\mu$ and $\delta$ is mainly a technical matter. In practice, they can hardly be disentangled in terms of the variables that make up social capability to assimilate spillovers. We mainly use the parameter $\mu$ to calibrate the model (i.e., to generate a setup that implies a reasonable borderline between catching-up and falling-

\footnotetext{
${ }^{3}$ To achieve this reaction of the spillover curve, the learning capability had to appear in two places in the spillover function (Equation 3).
} 
behind), while $\delta$ is used more actively in the simulation experiments below as an indicator of the learning capability of a region.

In order to be able to analyse the dynamics of convergence and divergence, we take the time derivative of the technology gap in Equation (4) and substitute equations (1), (2) and (3). For a two-region model this yields:

$$
\dot{G}_{i j}=\frac{d}{d t} \ln \frac{K_{i}}{K_{j}}=\frac{\dot{K}_{i}}{K_{i}}-\frac{\dot{K}_{j}}{K_{j}}=\frac{\alpha}{1-\alpha \beta \lambda}\left(\left(\rho_{i}-\rho_{j}\right)-\left(S_{j}-S_{i}\right)\right), \text { with } 0<\alpha \beta \lambda<1,
$$

in which $\alpha, \beta$ and $\lambda$ are assumed to have the same value in each region. This expression can be analysed using Figure 4.

We will restrict ourselves to describing only one case, namely the one in which region $i$ is the leader, i.e., where the initial gap is positive. We also assume that leadership implies larger $R \& D$ efforts, such that $\rho_{\mathrm{i}}>\rho_{\mathrm{j}} .{ }^{4}$ In Figure $4, \mathrm{~S}_{\mathrm{j}}-\mathrm{S}_{\mathrm{i}}$ represents the difference in received spillovers between the two regions. The lagging region receives positive net spillovers, as discussed above. Note that we have again assumed $\delta_{\mathrm{i}}=\delta_{\mathrm{j}}$ and $\mu_{\mathrm{i}}=\mu_{\mathrm{j}}$. In the more general case where these assumptions do not hold, the net spillover curve will not intersect with the origin, but this does not change the dynamics in a major way. The horizontal line $\rho_{\mathrm{i}}-\rho_{\mathrm{j}}$ displays the difference in the exogenous rate of growth of the knowledge stock between the two regions.

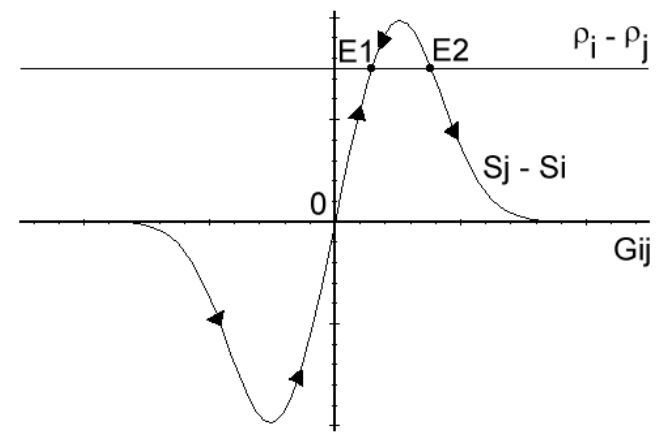

Figure 4

It is straightforward from equation (5) that when the curve in Figure 4 intersects with the horizontal line $\rho_{\mathrm{i}}-\rho_{\mathrm{j}}$, the time derivative of the technology gap is equal to zero. In other words, the intersection points correspond to equilibrium points. The (leftmost) intersection point at which the S-curve has a positive slope is stable, whereas the other intersection point is unstable. Thus, what happens to the knowledge gap in the long run depends on where the process starts. Starting points to the left of E2 will yield convergence to a stable technology

\footnotetext{
${ }^{4}$ This assumption is not essential. Obviously, the case where region $j$ is the leader is the mirror-image of the case we discuss.
} 
gap (corresponding to E1). Starting values to the right of E2 will yield falling behind, with an ever growing knowledge gap. ${ }^{5}$

Now consider what happens with changing parameter values. We will first consider a variation in the difference in the exogenous rate of growth of the knowledge stock between the two regions, $\rho_{i}-\rho_{j}$. If the difference is enlarged in favour of the leader, the $\rho_{i}-\rho_{j}$ line in Figure 4 moves upward, meaning that the range of technology gaps at which catch-up occurs becomes smaller. Eventually, when the $\rho_{i}-\rho_{j}$ line shifts to a position above the net spillover curve, there will be no opportunity at all for catch-up. If, on the other hand, the exogenous rate of growth of the knowledge stock in the backward region is increased (e.g. by expanding research efforts) up to a level comparable with the advanced region, i.e., the $\rho_{i}-\rho_{j}$ line ultimately coincides with the horizontal axis, and the (stable) equilibrium gap is zero, implying complete converge in the long run.

Next, we consider the impact of the geographical distance between the two regions. A decrease in the geographical distance has the effect that the spillover curves $S_{i}$ and $S_{j}$ increase proportionally to the decrease in geographical distance (explained by Figure 2) and the maximum of the $S_{j}-S_{i}$ curve in Figure 5 moves upwards ${ }^{6}$. Figure 6 displays the bifurcation diagram for this case. ${ }^{7}$ The horizontal axis of the bifurcation diagram shows the values of the geographical distance parameter $\gamma_{\mathrm{ij}}$. The vertical axis shows the equilibrium values of the technology gap. The line $\mathrm{E}_{\mathrm{sj}}$ shows the stable equilibrium, while the line $\mathrm{E}_{\mathrm{uj}}$ points to the unstable equilibrium. The line $S_{\operatorname{maxj}}$ represents the top of the net spillover curve in Figure 5 . This figure shows that for high values of $\gamma_{i j}$ no equilibrium value of the technology gap exists. In terms of Figure 5, this occurs when there are no intersection points between the curves. For a threshold value of $\gamma_{\mathrm{ij}}$, one equilibrium appears. This occurs when the two curves in Figure 5 are tangent. For values of $\gamma_{\mathrm{ij}}$ smaller than the threshold level, two equilibria exist, as described by the curves in the bifurcation diagram.

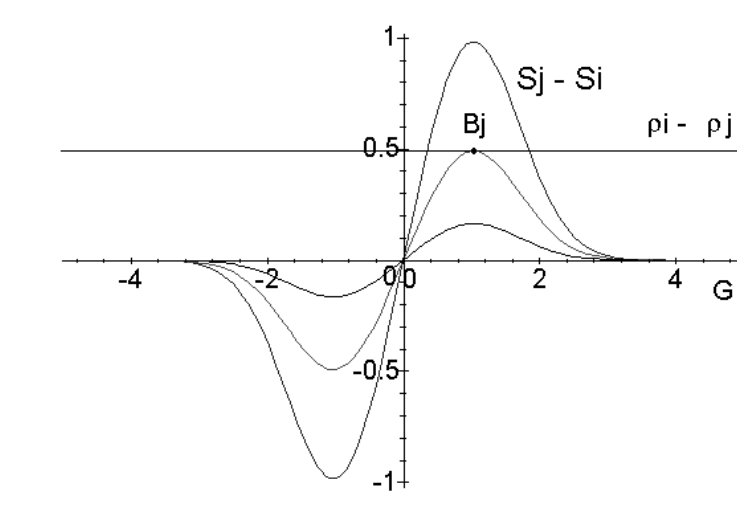

Figure 5

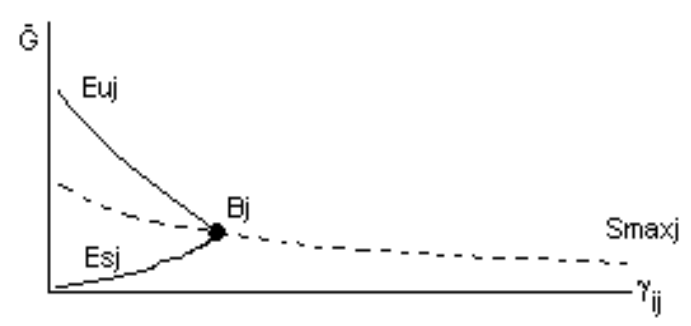

Figure 6

\footnotetext{
${ }^{5}$ Verspagen (1991) estimates a simpler version of this model for a large sample of countries over the post-war period, and finds that falling behind is a frequent phenomenon.

${ }^{6}$ The maximum also moves a little bit away from the y-axis, but this is a very small effect.
} 
A similar bifurcation analysis can be performed for the parameter $\delta$. The effect of an increase in the learning capability of the backward region $j\left(\delta_{\mathrm{j}}\right)$ on the $\mathrm{S}_{\mathrm{j}}-\mathrm{S}_{\mathrm{i}}$ curve is displayed in Figure 7. Note that $\delta_{\mathrm{j}}$ is the only parameter that has changed, $\delta_{\mathrm{i}}$ is kept constant. It can clearly be seen that on the right hand side of the figure the top of the curve has moved to the upper right of the figure and the curve does not intersect with the origin anymore. What has happened on the left-hand side is a bit more difficult to see. The minimum point has moved

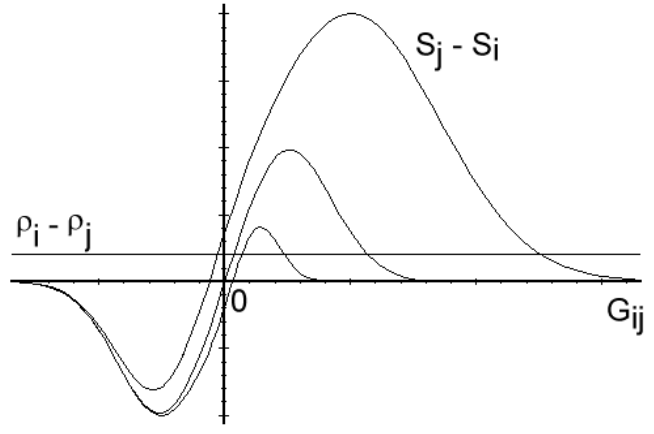

Figure 7

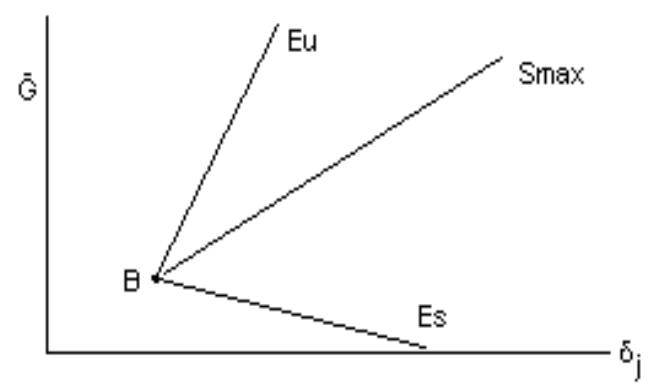

Figure 8

upwards so that it is closer to the horizontal axis. Also, there is a small movement of the minimum point away from the y-axis. The bifurcation diagram now looks as displayed in Figure 8.

Note that the $E_{s}$ line for the stable equilibrium can even go below the $x$-axis if the difference in exogenous growth rates of the knowledge stock is small enough, which illustrates an interesting special case of the model. This situation indicates a take-over in leadership by the (initially) lagging region. In terms of Figure 7, this occurs when the horizontal line $\left(\rho_{i}-\rho_{j}\right)$ intersects with the $S_{j}-S_{i}$ curve left from the y-axis, where the gap is smaller than zero, indicating that region $j$ is the leader region. The combination of a large learning capability in the lagging region together with a small difference in the exogenous rate of growth between laggard and leader gives rise to a take over of the lead position by the backward region. Note that it is primarily learning capability that drives this process of takeover.

We omit the bifurcation analysis for the $\mu$ parameter, which is relatively straightforward, and jump to extend the model to a multi-regional case. Suppose we have a world with $k$ regions, so that each region can be characterised by $k-1$ technology gaps (we omit the trivial case of $G_{i i}$ ). Spillovers are received from each of the other regions, so that the $S$ terms in equation (2) now become sums of spillovers over $k$ - 1 regions. This gives rise to the following modified form of equation (5):

\footnotetext{
${ }^{7}$ Note that the figure should show a discontinuous graph (in the model a geographical distance is either 1 or 2 , not 1.5), however, for visual reasons the individual points are connected.
} 


$$
\begin{gathered}
\dot{G}_{i j}=\frac{d}{d t} \ln \frac{K_{i}}{K_{j}}=\frac{\dot{K}_{i}}{K_{i}}-\frac{\dot{K}_{j}}{K_{j}}=\frac{\alpha}{1-\alpha \beta \lambda}\left(\left(\rho_{i}-\rho_{j}\right)+\left(\Sigma_{n} S_{i n}-\Sigma_{n} S_{j n}\right)-\left(S_{j}-S_{i}\right)\right), \\
\text { with } 0<\alpha \beta \lambda<1 \text { and } n \neq i, j,
\end{gathered}
$$

in which $\Sigma_{n} S_{i n}$ and $\Sigma_{n} S_{j n}$ denote the spillovers received by region $i$ and $j$ respectively from all regions $n$ for which $n \neq i, j$ (this term is thus invariant to $G_{i j}$ ). Note that equation ( $5^{\prime}$ ) specifies the (growth of the) gap between the two regions $i$ and $j$ only. There are $k$ regions in total, thus every region $i$ has $k-1$ of these equations.

Equation (5'), under the ceteris paribus assumption with respect to the knowledge stocks in regions other than $i$ and $j$, gives rise to identical figures as Figures 4-8. The only difference is that in the case of equation $\left(5^{\prime}\right),\left(\rho_{i}-\rho_{j}\right)$ and $\left(\Sigma_{n} S_{i n}-\Sigma_{n} S_{j n}\right)$ are lumped together into the horizontal lines that used to be determined by $\left(\rho_{i}-\rho_{j}\right)$ only. A movement of this horizontal line (and therefore in the horizontal position of E2) can now be caused by two factors. First, a variation in the difference between the exogenous rates of growth of the knowledge stocks of two regions (as before), and, second, a difference across regions in the spillovers received from all other regions.

The latter term is largely determined by geographic location. The subset of regions to which this term refers does not differ between $i$ and $j$, but when, for example, region $i$ is closer to the advanced regions than region $j$ is, this gives region $i$ an advantage over region $j$. Also, the learning capability $\left(\delta\right.$ and $\mu$ ) has an impact on how $\left(\Sigma_{n} S_{i n}-\Sigma_{n} S_{j n}\right)$ differs between $i$ and $j$ ).

\section{The influence of geographic distance on regional growth differentials}

When we specify a (symmetric) matrix of distances between regions, the model is fully specified, and time paths for the $G$ variables result from any set of initial values. However, for more than two regions, these time paths are extremely tedious to work out analytically, which is why we resort to simulations to describe the outcomes of the model. By carrying out many simulations (with randomised initial conditions) it is possible to examine the general behaviour of the model, and we find that certain patterns in the gaps of the knowledge stocks appear repeatedly. All simulations use a Pascal computer program that implements a RungeKutta algorithm to numerically solve the differential equations for $G$.

We use three different geographical spheres (distance matrices). These are a lattice of honeycombs, a column, and a globe. Appendix A gives an exact description and a map of these spheres. The regions on these spheres are assumed to be homogeneous areas. In other words, no differences of the relative importance (e.g., political) of the regions are assumed, nor do we assume differences in the degree of connectedness (e.g. the presence of harbours, mountains, roads and railways). Since this is a one-sector model, we also assume that the regions have homogenous economic structures. The first of these spheres is two-dimensional. A honeycomb pattern is chosen in order to provide an equal amount of contingent neighbours for each region, with each neighbour having an equally long border. This would not be the case when using a lattice of squares, which would have the additional difficulty of judging the 
importance of the different kinds of neighbours - queens, bishops or rooks ${ }^{8}$ - by assigning weights to them. Because the lattice is flat and has a hexagonal shape in itself, there is always exactly one central region. This region has a favourable location, as will become clear from the experiments.

The second sphere used has the shape of a column. In the column, the single central region from the lattice is replaced by a belt of regions around the middle of the column. Finally, a globe is modelled. In the globe, no inherently central location is present. Both the column and the globe consist of a honeycomb pattern, in the case of the globe pentagons had to be added (the regions are constructed as the pattern on a soccer ball, i.e., 12 pentagons and 20 hexagons $)^{9}$. The lattice of honeycombs can be considered similar to a country, whereas the globe could be a model for a world. The column can be seen as an intermediate case, since it replaces the one central region of the lattice by a belt of centrally located regions, whereas on the globe every region is centrally located.

Geographic distance in the three geographical spheres is measured by assigning a weight of 1 to neighbouring regions (in the sense that two regions share one border). Regions which do not share a border with a specific region are given a weight by using the concept of nearest neighbours, which means that a different (lower) weight is attributed to a second order neighbour. A second order neighbour does not share a border with a specific region, but does share a border with a neighbour of the specific region. Thus, no evaluation of relative importance of the connection between regions, based on ex ante known information (for example the presence of roads and railways) is taken into account. Only geographical distances are reflected. In this way, the distance $d_{i j}$ is determined for every region towards every other region. Now, it is possible to construct a region-by-region matrix of shortest paths. Then, the corresponding weights $(\gamma)$ are determined using the inverse of the orders (inverse shortest path, Hagett, Cliff and Frey, 1977). Note that this way of measuring geographical distance is a special case of $1 /\left(d_{i j}{ }^{x}\right)$ with $x$ equal to 1 .

Our first simulation experiment assumes that all regions are initially equal, except for their geographic location ${ }^{10}$. Thus, $\delta_{\mathrm{i}}=\delta, \mu_{\mathrm{i}}=\mu$, and initially all values for $G$ are equal to zero. The

\footnotetext{
${ }^{8}$ These terms are borrowed from chess. A queen is allowed to move in all directions indicating that all 8 neighbours of a square are equally important. A lattice with these characteristics is called a Moore neighbourhood. A bishop is only allowed to move in a diagonal way, while a rook is only allowed to move horizontally or vertically, meaning that one might want to assign a different (lower) weight to a neighbours, which do not share a border but only one point (the bishops-case) than to neighbours, which do share a border (the rooks-case). When only neighbours of the rook type are considered, the plain is called a von-Neumann neighbourhood.

${ }^{9}$ It is impossible to construct a three-dimensional figure by the single use of hexagons. Hexagons will always produce a flat sphere, since the sum of the angles of three contingent hexagons is equal to 360 degrees. By adding pentagons, the total angle will be less than 360 and thus producing a three-dimensional figure. It would have been possible to construct a three-dimensional sphere by using pentagons only, however, in that case the total number of pentagons (regions) used would be twelve. The globe that is used in the simulations consists of thirty-two planes (regions), which was considered to give more interesting interactions than a sphere containing only twelve planes.

${ }^{10}$ The initial values of the data are described in Appendix B.
} 

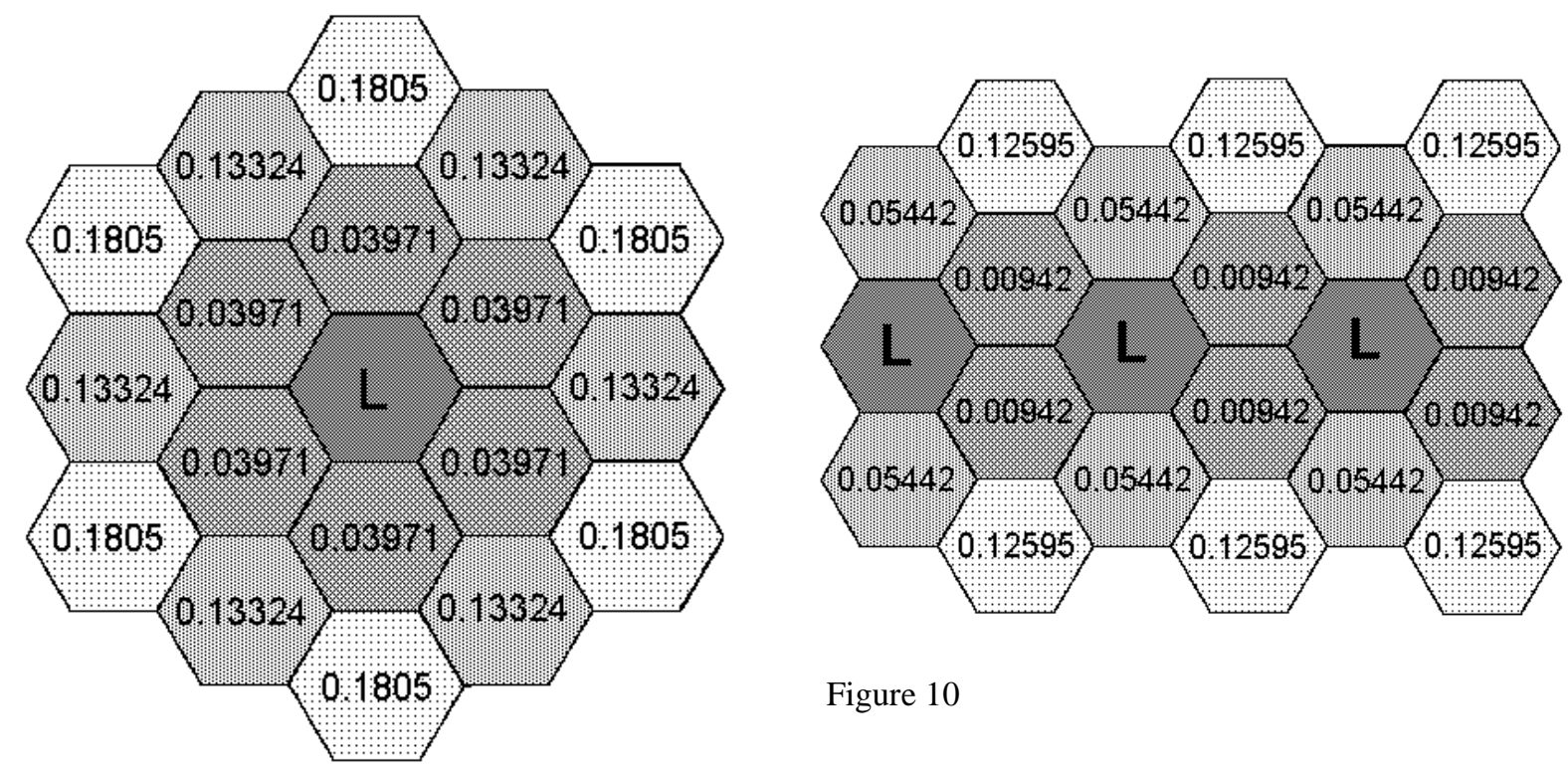

Figure 10

Figure 9

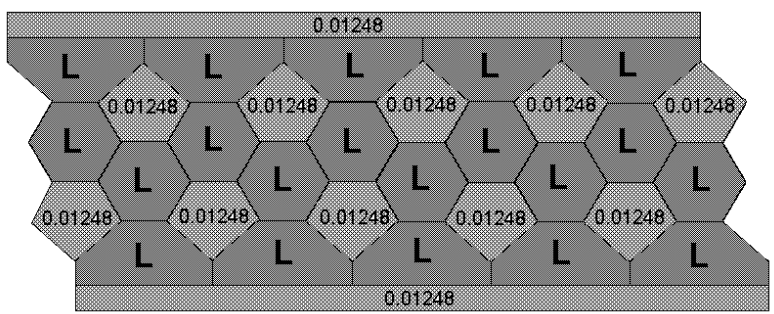

Figure 11

values for the parameters $\delta$ and $\mu$ remain fixed over the run, while the values for $G$, naturally, evolve according to equation (5'). Figures 9, 10 and 11 show the resulting distribution of the gaps (after 10000 simulation periods) toward the leader of each region in the different geographic spheres. ${ }^{11}$

Figure 9 displays the resulting pattern for the lattice of honeycombs. The numbers within each cell refer to the size of the technology gap a region has towards the leader region. Darker patterns correspond to smaller gaps. Knowledge spills into to a region more easily when the distance towards a knowledge intensive region is small. Since the central region has the most favourable location for receiving knowledge spillovers, it will end up being the leader.

Figure 10 displays the resulting gaps for the column. The regions located in the middle band of the column have the most favourable position with respect to receiving spillovers from neighbouring regions, because they have more neighbours with a low order than the regions on the edge of the column. These three regions therefore come out as the joint leaders.

Finally, Figure 11 displays the results for the globe. In this sphere, all hexagon shaped regions have an equal potential for receiving knowledge spillovers. The same holds for all

\footnotetext{
${ }^{11} 10000$ periods is always enough to let the gaps reach their equilibrium values, i.e., no change has occurred for a long time when the system reaches period 10000 .
} 
pentagon-shaped regions. However, a hexagon-shaped region has a small advantage over a pentagon-shaped region with respect to receiving knowledge spillovers, since the honeycombshape provides a region with six instead of five direct neighbours and thus probably more spillovers. Figure 11 indicates that this is indeed the case, all hexagon-shaped regions have a leading position, while all pentagon-shaped ones display a small gap.

Summarising, the lattice shows a typical resulting pattern with the centrally located region being the leader (i.e., a strong polarisation of economic growth). The column is characterised by a belt of leaders. The globe has many leaders, since all hexagon-shaped regions have the same favourable geographic location for receiving spillovers from other regions.

These three figures have shown the pattern of gaps that is solely determined by geographical distances. Next, two sets of experiments have been set up in which systematic differences between regions are introduced. The first set considers a variation across regions of the intrinsic learning capability parameter $\delta$. In the second set, the exogenous rate of knowledge generation is varied across regions.

\section{Systematic differences in the learning capability parameter $\delta$}

In this set of experiments, a randomly generated value for $\delta$ is assigned to each region. The value for $\delta$ is drawn from a uniform distribution with upper boundary 2, and lower boundary varying from 1.8 until 2 , taking steps of 0.01 . The choice of these ranges is based on the calibrations of the model. In none of these ranges falling behind occurs, i.e., an equilibrium value of the gap always exists. Fifty runs were done for each range. Recall that the higher $\delta$ for a region, the more it is capable to implement the knowledge that spills over from other regions.

Figures 12, 13, and 14 show the results of this experiment for each geographical sphere. The horizontal axis displays the lower boundary of the range out of which the learning capabilities are assigned. As we move from left to right on this axis, the range from which values for $\delta$ are drawn becomes smaller, and hence (on average) the regions become more equal with respect to their value of $\delta$. On the extreme right hand side, the uniform range reduces to a single value (i.e., 2), and we are back in the situation where all parameters are equal (Figures 9-11). On the vertical axis, the coefficient of variation of the resulting technology gaps over all regions (after 10000 simulated time periods) is shown. The shades in the figure correspond to frequencies over the 50 runs, with complete black corresponding to a frequency of 50 (i.e., all runs). White shades indicate very low (sometimes zero) frequencies.

The sensitivity to the lower boundary for the lattice is shown in Figure 12. For large ranges, i.e., heterogeneous values for $\delta$, the outcome of the process as characterised by the coefficient of variation varies greatly. No unique value, or range of values, emerges as an attractor of the process. When the range from which we draw $\delta$ becomes smaller, i.e., when we move to the right in the figure, the process 'zooms into' a narrowing range for the coefficient of variation. Thus, the result for the lattice appears as comet-shaped. The trail of the 'comet' disperses equally to all directions as the lower boundary is decreased. 
For the column (Figure 13) and the globe (Figure 14) the same analysis is done. One striking difference as compared to Figure 12 emerges from these two figures. The coefficient of variation for the column is also comet-shaped, but the trail extends in a downward direction for larger ranges (i.e., moving to the left). The globe shows the same downwardly sloping trend (from right to left) as the column does. In this case, however, the band does not widen very much when moving right to left, i.e., the dispersion of results remains relatively constant.

Note that the result of a downward slope (moving right to left) is somewhat counterintuitive. It says that higher diversity across regions in terms of the learning capability parameter $\delta$ causes lower levels of disparity in the resulting gaps at the end of the simulation, whereas less diversity in $\delta$ leads to more disparity in knowledge stocks. Thus, compared to a situation of identical regions, introducing heterogeneity in terms of learning capability leads to convergence. Compared to the intuition from the convergence theory briefly sketched in the introduction, this is a surprising result.

What causes this surprising result? When the range is limited to only one value (in this case this value is 2), the only factor that has an influence on the difference between regions is the geographical distance. As was seen before (Figures 9-11). When differences with respect

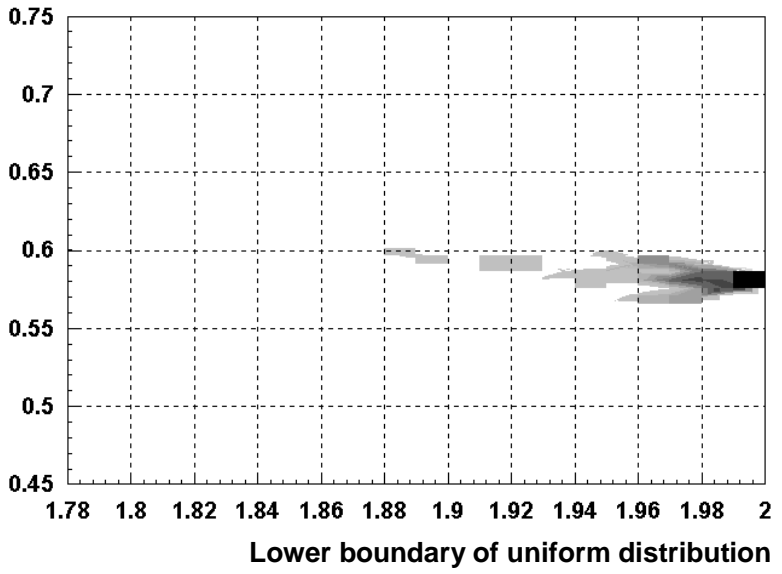

Figure 12: Frequency diagram of the coefficient of variation at the end of the run

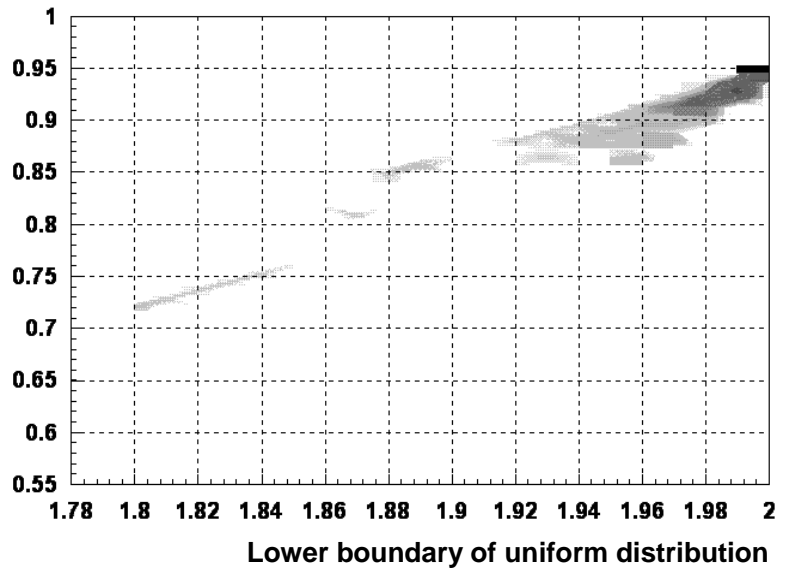

Figure 13: Frequency diagram of the coefficient of variation at the end of the run

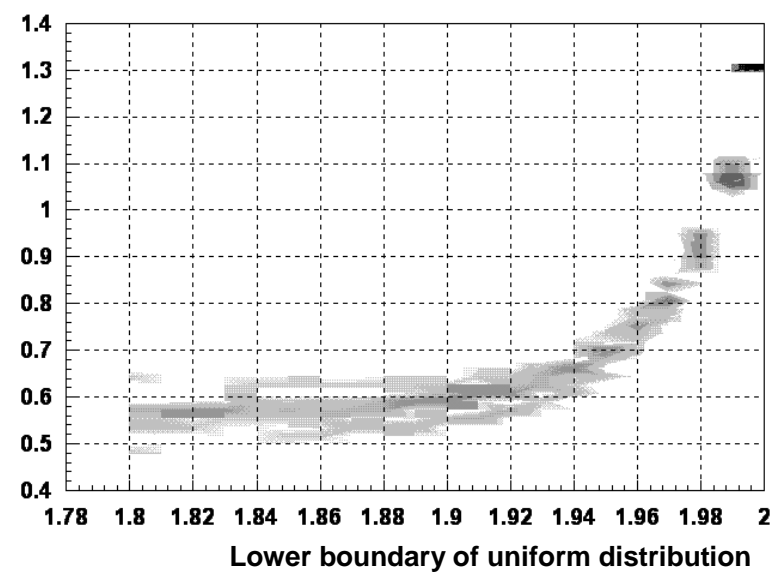

Figure 14: Frequency diagram of the coefficient of variation at the end of the run 
to $\delta$ are increased, a second polarising factor executes an influence on the regions. These two polarising factors counteract. On average, a number of regions with unfavourable geographical locations will randomly obtain a high learning capability, while some centrally located regions will randomly obtain a low learning capability. This leads to lower levels of disparity within one run, but also leads to more disparity across the runs. Since there is more opportunity for the learning capability as a second polarising factor with larger ranges (at the left end of the figure), the outcomes - in terms of coefficient of variation - of different runs become more distinct, causing the fan-shape in the lattice and the column.

This does not yet explain why the figures for the column (13) and globe (14) show this counterintuitive result, and the lattice does not. The difference between the spheres lies mainly in the fact that the column and globe have multiple geographically favoured regions. For the lattice there is only one central region, while the six first ring regions have a relatively large gap.

This means that in the case of a globe or column, there is more than one 'neighbourhood', with a 'local leader'. When such a 'local' leader is affected by a low learning capability (which is more likely for larger ranges of this parameter), this not only affects the local leader, but indeed the whole neighbourhood. This causes an overall lower coefficient of variation. On the other hand, a lower coefficient of variation could be the result of higher learning capabilities for the peripheral regions. However, the chance that all (or at least a large part of the) peripheral regions (randomly) obtain(s) a higher learning capability than the potential local leaders is limited. The impact of differences in learning capability grows disproportionally with the range, causing the upward sloping pattern in Figures 13 and 14. Since the globe has more potential local leaders than the column (20 versus 3 ), the chance is higher in case of the globe that a local leader (randomly) obtains a low learning capability. Therefore, Figure 14 displays a steeper upward slope than Figure 13.

What is also peculiar in Figure 14 is that from 1.9 downwards there seems to be a horizontal trend. This can be explained as follows. At the left-hand side of the figure, the randomness in the parameter is apparently so high that all central regions are affected, and additional randomness does not increase the chance that an additional potential local leader obtains a relatively low learning capability. Therefore, the number of potential local leaders does not decrease any further at larger parameter ranges, resulting in a horizontal trend in the figure.

The conclusion that can be drawn from these figures (13 and 14) is an extraordinary one. Contrary to what is generally assumed, it is found that when differences in learning capabilities increase (decrease) across regions, there may be a tendency for the disparity between them to decrease (increase). This is a direct result from the inclusion of geographical distance in the model. Thus, the results show that leaving out distance from a model of technology gaps may indeed affect the results.

One may ask the question as to whether or not this result is robust to considering variations in other parameters, i.e., $\mu$ and $\rho$. Limitations of space do not permit us to present results for 
both $\mu$ and $\rho$. We will shortly discuss the results for variations in $\rho$. Readers interested in variations in $\mu$, which yield similar patterns, may request these results from the authors.

\section{Differences in the exogenous rate of knowledge generation $\rho$}

In the following set of experiments the exogenous rate of growth of the knowledge stock is randomly chosen from different ranges, while the initial knowledge stocks (as well as other parameters) of the regions are identical across regions. In this experiment, it generally does matter for final leadership whether a region has an initial advantage. The region that becomes a leader early on will, in most of the cases, remain in this position. There are two determinants of leadership in the first stages of the simulation. First, the exogenous rate of growth of the knowledge stock is a very important influence in determining the leader in the first periods of simulation. Second, the geographical distance to centrally located regions is also important. In general, the region that has the highest $\rho$ among the centrally located regions will most likely become a leader.

In order to investigate the sensitivity of the model to changes in the range out of which the exogenous rate of growth was chosen, several sets of 50 runs were carried out each with a different range. Figures 15,16 , and 17 show the results when the lower boundary of the range

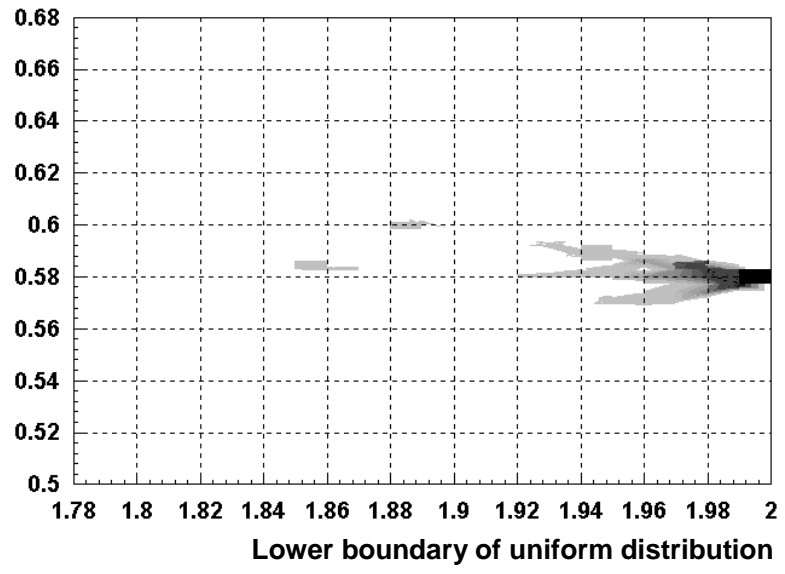

Figure 15: Frequency diagram of the coefficient of variation at the end of the run

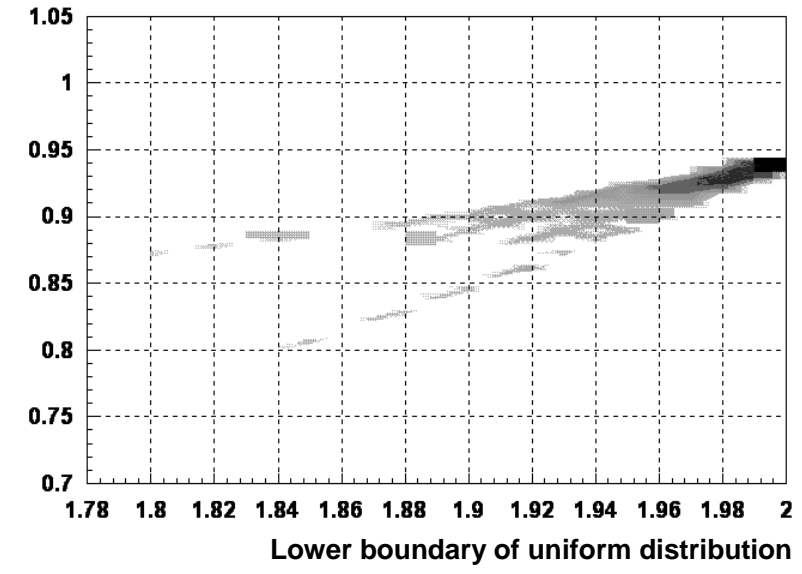

Figure 16: Frequency diagram of the coefficient of variation at the end of the run

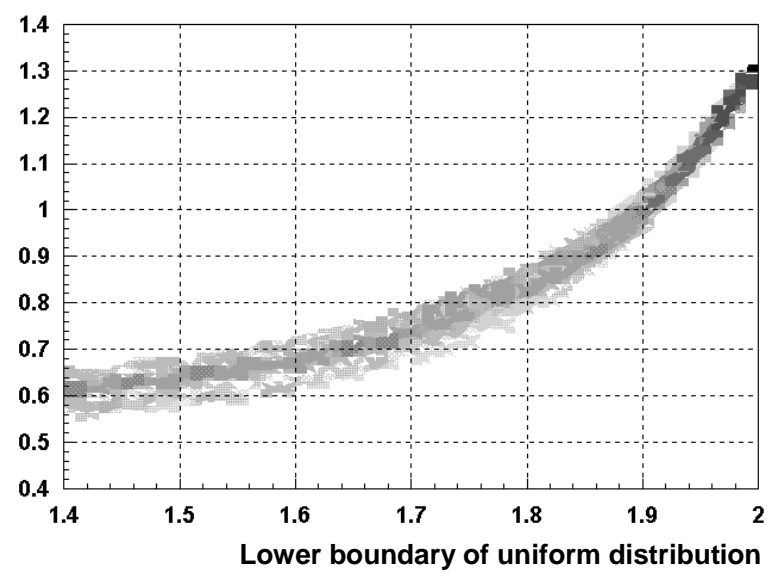

Figure 17: Frequency diagram of the coefficient of variation at the end of the run 
is increased from 1.8 to 2 with steps of 0.01 . The upper boundary is held constant at 2 . The vertical axis shows the coefficient of variation over the last period in every run.

For the lattice (Figure 15), the coefficient of variation displays a fan-shape (identical to the experiment with the learning capability). All coefficients of variation tend to one value as the gap between lower and upper boundary decreases. The explanation is the same as in the experiment with the learning capability. Increasing the difference in exogenous rates of growth of the knowledge stock introduces a second polarising factor, next to the geographic distance. The two polarising factors counteract, thereby causing a larger equality across gaps within one run and a larger disparity across coefficients of variation across several (50) runs.

For the column, the sensitivity to the lower boundary of the interval is given in Figure 16. Contrary to the lattice, this sphere seems to generate mostly coefficients of variation that are smaller than the one in which three leaders appear (the dark spot at the right of the figure) ${ }^{12}$. Again a fan-shape appears. The explanation for these features is the same as for the case in which the learning capability was varied across ranges. The fan-shape is generated by the growing inequality in the coefficient of variation across runs, as the difference in learning capabilities across regions counteracts the difference in geographic location (from right to left). The line is upward sloping because within each run the disparity across gaps increases, as the range is decreased, due to the characteristics of the column.

The figure for the globe (Figure 17) shows a curve that is upward sloping and shows less of a fan-shape. There is only little dispersion at the left-hand side of the curve. Contrary to the former experiment with the learning capability, this curve does not yet show a horizontal part when the gap between lower and upper boundary is large. However, we see that the lowest observation has a coefficient of variation of about 0.6 at a lower boundary of 1.4. If the lower boundary would further be decreased, the figure would start to show a horizontal trend.

The general conclusion from this section follows the same directions as the former section in which the learning capability of each region was varied. The patterns in this section show similar trends, although there are a few differences. The graph for the column under a variation of the exogenous rate of growth shows the same fan shape, but the upward trend is much less steep than under a variation in the learning capability in the former section. This indicates that a variation in the learning capability has a stronger counteracting effect (to geographical distance) than a variation in the exogenous rate of growth of the knowledge stock. If the range in which a parameter can vary is small, there exists more dispersion of the gaps across runs in the case of the learning capability compared to the exogenous rate of growth of the knowledge stock.

Virtually the same holds for the globe. The effect of the learning capability on overall disparity is larger than in the experiment in which the exogenous rate of growth of the knowledge stock was varied in the same range.

\footnotetext{
${ }^{12}$ Figure 7 shows the distribution of the gaps across regions as occurs at the utter right of the figure.
} 


\section{Conclusions}

This paper presented a model for knowledge spillovers based on geographical distance as well as technological distance. The regions in our model receive knowledge spillovers from other regions, and this enables them to grow rapidly. Our model is similar to some of the models found in the 'technology gap' tradition of analysing convergence of GDP per capita. Compared to these models, we add the spatial distance effect on spillovers. The further away other regions are, the less strong spillovers from these regions are. Our research question is what is the effect of including spatial distance on convergence. From the simulations, we can draw several conclusions. When regions are located on a two-dimensional lattice, the more differences there are across regions with regard to learning capability or the exogenous rate of growth of the knowledge stock, the larger is the variation in resulting gaps. The runs can show both higher and lower disparity than the 'perfect' distribution of gaps as regions initially have more differences.

For a column-shaped geographical structure, the case in which all regions are equal to each other generates the highest disparity. As soon as regions show differences with respect to the learning capability or the exogenous rate of growth, the disparity becomes less. Contrary to the situation for the lattice, there are no runs that have a larger coefficient of variation than the case with identical regions.

When regions are grouped on a globe, which has many central regions as compared to the column, a slightly different effect of the change in parameters is found. As with the column, the disparity decreases as regions show larger differences with respect to the value of the parameter. However, the slope is non-linear, and for a certain minimum level of disparity, the disparity will not decrease any further. Larger initial differences in parameters will induce results, which have a similar coefficient of variation.

This result stems from the process in which varying a parameter such as the learning capability or the exogenous rate of knowledge generation, provides a counteracting force relative to the effect of spatial distance. Increasing differences in learning capability or the (exogenous) rate of growth of knowledge $(R \& D)$ are in themselves polarising factors, leading to the tendency that regions with high values for these parameters grow rapidly. The spatial structure on which regions are grouped is also a polarising factor, leading, ceteris paribus other parameter differences, to high growth rates in centrally located regions. Combining differences in learning capability or exogenous knowledge growth with spatial distance leads to the result that the two forces counteract each other. 


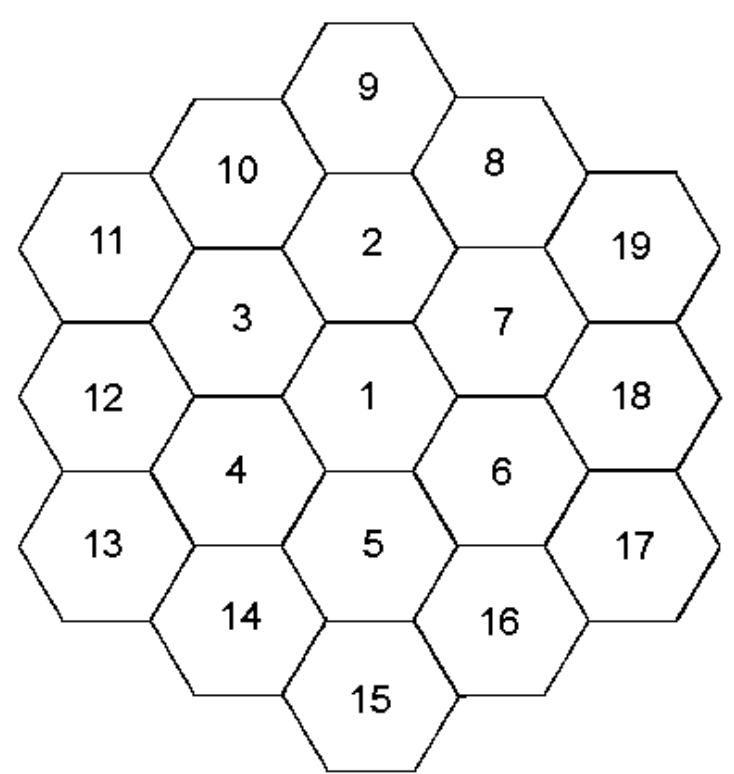

Figure A-1: Lattice of honeycombs

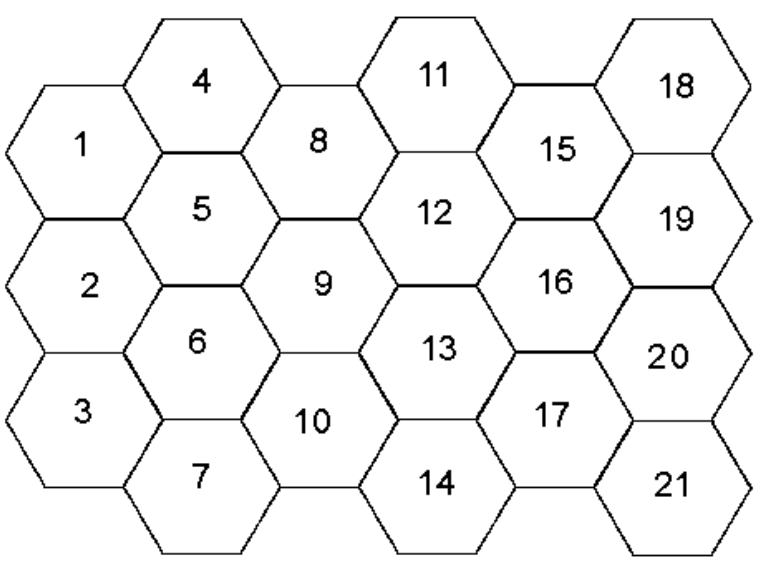

Figure A-2: A column

Figure A-1 displays the topography of the regions on a lattice of honeycombs. The number within each hexagon was used to establish the geographical distances between all hexagons. Figure A-2 displays the column. Region 1 borders to regions 4,5 and 2 as well as 18 and 19, region 2 to regions 1, 5, 6 and 3 as well as 19 and 20, and region 3 has next to region 2, 6 and 7 also regions 20 and 21 as its direct neighbours. Thus, if one would walk from region 20 to the right, one would reappear at the left of the figure in region 2 or 3.

The figure below (Figure A-3) represents a globe with 12 pentagons and 20 hexagons. For the graphical representation, we used the same principle that was applied in making a map of the world. Hence, the regions close to the poles look larger as they actually are, while the regions around the equator show their true proportions. At the bottom and at the top are regions 29 and 9. These are pentagons, for example region 9 borders to five regions, namely 3,2, 8, 10 and 11. Region 29 and 9 are in reality as large as region 1 . The graphic representation of a globe has also as a consequence that for example region 3 seems to differ in size from region 6. Again, this is not the case in reality, region 3 is an ordinary hexagon. The same goes for all the other regions bordering 9 or 29. It should also be noted that region 11 borders not only to regions 9, 10, 24, 25 and 12, but also to region 3. In this way, region 12 also borders to regions 3 and 4, region 13 has regions 4 and 14 as direct neighbours as well, whereas region 28 also shares a border with regions 14 and 15 .

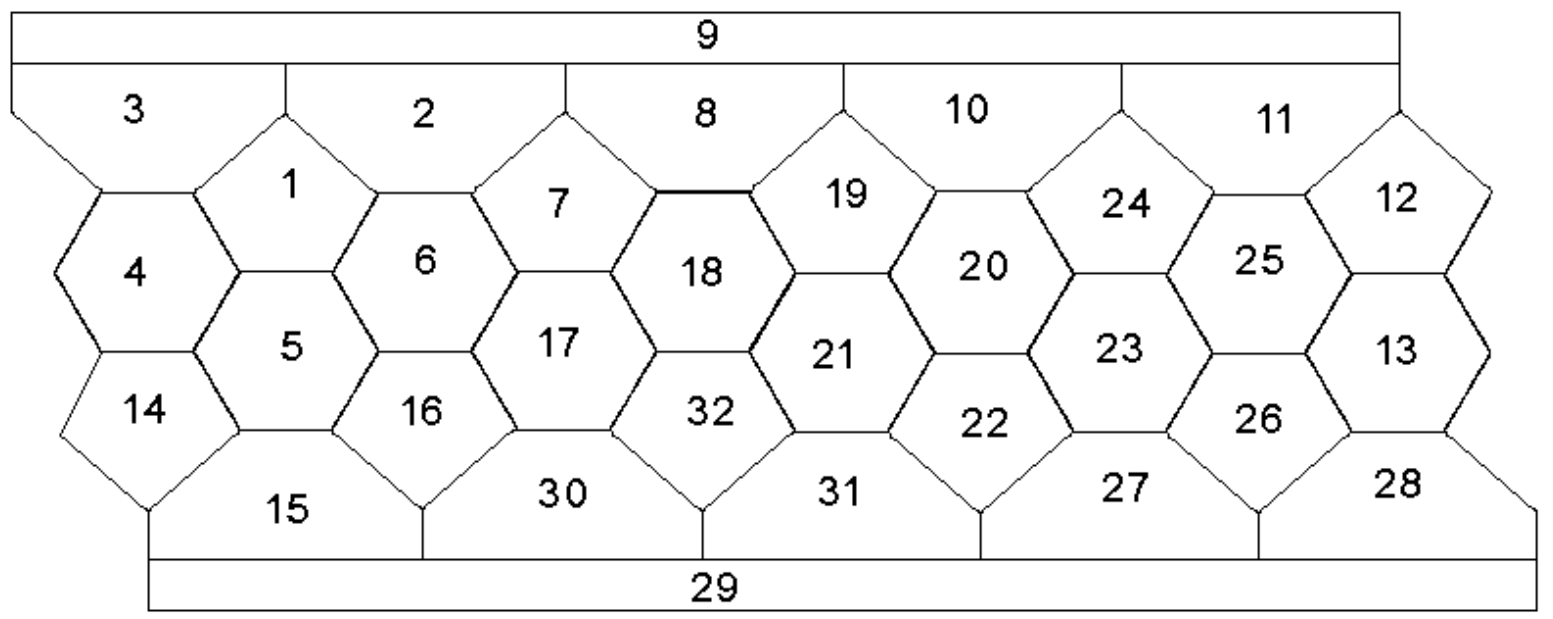

Figure A-3: A globe 


\section{APPENDIX B}

Default levels of the variables and values of the parameters:

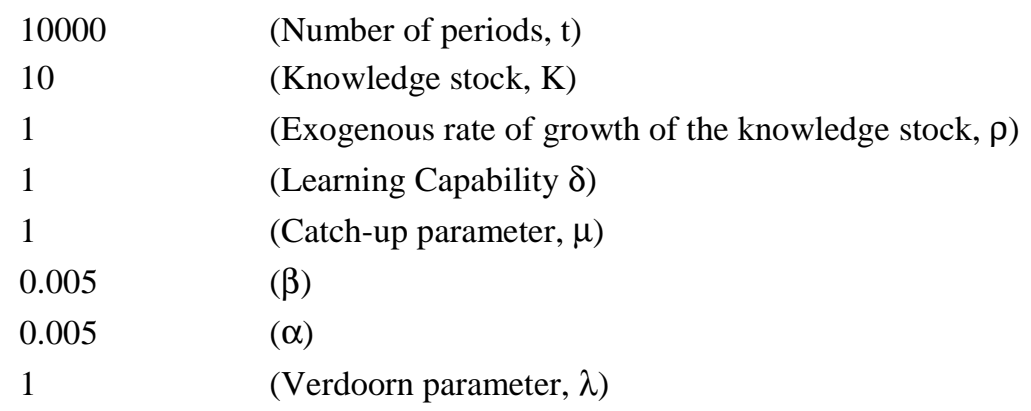

$\gamma$ (geographical distance) is constructed with the help of three different types of distance tables, one for each sphere.

\section{References}

Abramovitz, M.A., (1994), 'The Origins of the Postwar Catch-Up and Convergence Boom', in: Fagerberg, J., Verspagen, B. and N. Von Tunzelmann (eds), The Dynamics of Trade, Technology and Growth, Aldershot: Edward Elgar, pp. 21-52.

Acs, Z.J., Audretsch D.B. and M.P. Feldman, (1992), 'Real effects of academic research: comment', American Economic Review, vol. 82(1), pp. 363-367.

Amable, B., (1993), 'Catch-up and convergence: a model of cumulative growth', International Review of Applied Economics, vol. 7(1), pp. 1-25.

Audretsch D.B. and M.P. Feldman, (1996), 'Knowledge spillovers and the geography of innovation and production', American Economic Review, vol. 86(3), pp. 630-640.

Baptista, R., (1998), 'Clusters, innovation and growth: a survey of the literature', in Swann, P.G.M., (ed.), The dynamics of industrial clustering - international comparisons in computing and biotechnology, Oxford: Oxford University Press, pp. 13-51.

Barro, R.J., (1984), Macroeconomics, New York: Wiley.

Barro, R.J. and X. Sala-i-Martin, (1991), 'Convergence across states and regions', Brooking Papers Economic Activity, vol. 1, pp. 107-182.

Barro, R.J. and X. Sala-i-Martin, (1992a), 'Convergence', Journal of Political Economy, vol. 100, pp. 223-251.

Barro, R.J. and X. Sala-i-Martin, (1992b), 'Regional growth and migration: A Japan-United States comparison', Journal of the Japanese and International Economies, vol. 6, pp. 312346.

Barro, R.J. and X. Sala-i-Martin, (1995), Economic growth, New York: McGraw-Hill.

Baumol, W., (1986), 'Productivity growth, convergence and welfare: What the long-run data show', American Economic Review, vol.76, pp. 1072-1085. 
Breschi, S., (1995), 'Spatial patterns of innovation: evidence from patent data', paper presented at the workshop on 'New Research Findings: The Economics of Scientific and Technological Research in Europe', Urbino, Italy, 24-25 February 1995.

De Long, J.B., (1988), 'Productivity growth, convergence and welfare: comment', American Economic Review, vol.78, pp. 1138-1159.

Fagerberg, J., (1988), 'Why growth rates differ', in Dosi, G. et al. (eds), Technical change and economic theory, London: Pinter Publishers, pp. 432-457.

Fagerberg, J., (1994), 'Technology and international differences in growth rates', Journal of Economic Literature, 32, pp. 1147-1175.

Hagett, P., A. D. Cliff and A. Frey, (1977), Locational models, London: Edward Arnold (publishers) Ltd.

Jaffe, A., (1989), 'Real effects of academic research', American Economic Review, vol. 79, pp. 957-970.

Jaffe, A., Trajtenberg, M. and R. Henderson, (1993), 'Geographic localization of knowledge spillovers as evidenced by patent citations', Quarterly Journal of Economics, vol. 108, pp. 577-598.

Freeman, C., (1991), 'Networks of innovators: a synthesis of research issues', Research Policy, vol. 20, pp. 499-514.

Malerba, F., and L. Orsenigo, (1995), 'Schumpeterian patterns of innovation', Cambridge Journal of Economics, vol. 19(1), pp. 47-66.

Pavitt, K. (1987), On the nature of technology, Brighton: University of Sussex - Science Policy Research Unit.

Verspagen, B., (1991), 'A new empirical approach to catching up and falling behind', Structural Change and Economic Dynamics, vol. 2, pp. 359-380.

Von Hippel, E., (1988), The sources of innovation, New York: Oxford University Press.

Von Hippel, E., (1994), “Sticky information" and the locus of problem solving: implications for innovation', Management Science, vol. 40(3), pp. 429-439. 